\title{
Diagnosis and classification of inflammatory bowel disease
}

\author{
Martin Fishman MD FRCP
}

\begin{abstract}
ARTICLES
Dubinsky MC, Ofman JJ, Urman M, Targan SR, Seidman EG. Clinical utility of serodiagnostic testing in suspected pediatric inflammatory bowel disease. The American Journal of Gastroenterology 2001;96:758-65.
\end{abstract}

Bezabeh T, Somorjai RL, Smith ICP, Nikulin AE, Dolenko B, Bernstein CN. The use of ${ }^{1} \mathrm{H}$ magnetic resonance spectroscopy in inflammatory bowel diseases: distinguishing ulcerative colitis from Crohn's disease. The American Journal of Gastroenterology 2001;96:442-8.

\section{ARTICLE SUMMARIES}

In the study by Dubinksy et al, 128 consecutive pediatric patients presenting to a major pediatric hospital with possible inflammatory bowel disease (IBD) were evaluated in standard fashion, and with antineutrophil cytoplasmic antibodies (ANCA) and anti-Saccharomyces cerevisae antibody (ASCA) testing by fixed ELISA ('modified assay'); positive ANCA specimens were subjected to direct immunofluorescence ('traditional assay') to determine the predominance of perinuclear or cytoplasmic staining pattern. Once a final diagnosis of IBD was established, the sensitivity, specificity and predictive value of serodiagnostic screening were determined. A sequential testing strategy was then developed consisting initially of the sensitive modified assay followed by the more specific traditional assay. This approach correctly identified $95 \%$ of non-IBD patients, who were spared unnecessary invasive investigation. Of IBD patients, 69\% were correctly identified. By applying sequential testing, rather than the modified assay alone, the absolute reduction of false-positive results was $13 \%$.

In the study by Bezabeh et al, colonic mucosal biopsies from 45 patients with ulcerative colitis (UC) and 31 patients with Crohn's disease (CD) were subjected to ${ }^{1} \mathrm{H}$ magnetic resonance spectroscopy (MRS) and multivariate analysis, and typical spectral patterns for these diseases were described. When the derived algorithm was applied blindly to specimens, the classification accuracy was $98.6 \%$. For distinction of IBD from normal controls, accuracy was $97.9 \%$. When applied to histologically normal colonic biopsies from IBD patients, 34.2\% showed abnormal MRS spectral patterns and another $18.4 \%$ could not be unambiguously classified. The spectral regions with the highest discriminatory potential were for choline, creatine, lysine, lipid and amino acids.

\section{COMMENTARY}

UC and CD can usually be distinguished on the basis of global evaluation of clinical, radiographic, endoscopic and histological features. However, when inflammation is limited to the colon, approximately $5 \%$ to $10 \%$ of patients cannot be definitively classified as having $\mathrm{UC}$ or $\mathrm{CD}$ by these usual means, and are given the default diagnosis of 'indeterminate colitis' (1). Patchiness of disease, once thought to be specific for $\mathrm{CD}$, is now recognized in $\mathrm{UC}$, especially when partially treated, and even rectal sparing may occur (2). The distinct diagnosis of UC or CD may be of great importance to the patient because these entities respond differently to specific therapeutic agents such as infliximab, may carry different risks for colon cancer, and may have serious implica- 
tions for surgery; pelvic pouch-anal anastomosis is a viable option for many patients with UC but is not generally recommended for patients with CD. Attention has recently focused on serodiagnostic markers as a means of distinguishing UC from CD in patients with indeterminate colitis $(3-5)$.

Two antibodies have received the most attention: ANCA (ANCA with perinuclear staining characteristics [pANCA]) and ASCA. pANCA is present in approximately $60 \%$ to $70 \%$ of patients with UC and $10 \%$ of patients with $\mathrm{CD}$, and the numbers are reversed for ASCA. A combination of these markers may be helpful in correctly classifying the majority of patients with indeterminate colitis (4). However, there will still be patients who cannot be definitively diagnosed.

The articles summarized here, appearing in consecutive issues of The American Journal of Gastroenterology, further expand our knowledge in these areas. Marla Dubinsky et al employed serological markers as an initial screening test for pediatric patients presenting with possible IBD when the diagnosis is uncertain. This approach seems helpful in selecting patients for more invasive confirmatory investigations, and may spare children with functional disorders from unnecessary diagnostic procedures. In the pediatric population, the clinical diagnosis of IBD may be more obscure than in adults, and serodiagnostic markers may not be as useful in screening adults presenting with nonspecific intestinal symptoms. This would require further study. The authors caution that their results may not be applicable to the nontertiary setting, but I believe that children should not be subjected to colonoscopy except in centres with expertise in managing pediatric gastrointestinal disorders.

In an altogether different vain, Tedros Bezabeh and others, collaborating with Charles Bernstein in Winnipeg, developed a novel approach to differentiating CD from UC. MRS, with multivariate analysis of spectral data, can distinguish these diseases with approximately $98 \%$ accuracy. Typical spectral patterns have been described for the two main types of IBD, and correctly classify patients with robustness. Moreover, mucosal regions that are not grossly or histologically inflamed may show MRS abnormalities that may represent tissue that is subclinically or preclinically 'at risk, ie, tissue that may be targeted for the subsequent development of inflammation. Simple endoscopic mucosal biopsies are required, but it is not clear whether MRS is available or practical in most centres. The article is technical, and elaborates on the methods in sophisticated detail. Patients with indeterminate colitis were not included in this study, so we are not yet able to apply this approach to this group confidently. Because these patients potentially have the most to gain from accurate classification, I hope we will see further data in this direction.

The serodiagnostic IBD markers may have other uses. For example, pANCA in patients with CD may indicate a clinical subtype with UC-like features $(5,6)$. In patients with UC, it may predict a higher likelihood of primary sclerosing cholangitis (7), aggressive left-sided disease (8) or postcolectomy pouchitis (9), which indeed is predisposed by the presence of primary sclerosing cholangitis. However, it is not known, for example, whether it may indicate patients at higher risk for dysplasia and malignancy. The ultimate clinical utility of these tests will also depend on availability, which is currently not widespread. Meanwhile, in the field of IBD classification, it is clear that our Canadian colleagues are helping illuminate the path ahead.

\section{REFERENCES}

1. Price AB. Overlap in the spectrum of non-specific inflammatory bowel disease "colitis indeterminate." J Clin Pathol 1978;31:567-77.

2. Bernstein CN, Shanahan F, Anton PA, et al. Patchiness of mucosal inflammation in treated ulcerative colitis: A prospective study. Gastrointest Endosc 1995;42:232-7.

3. Quinton JF, Sendid B, Reumaux D, et al. Anti-Saccharomyces cerevisiae mannan antibodies combined with antineutrophil cytoplasmic autoantibodies in inflammatory bowel disease. Prevalence and diagnostic role. Gut 1998;42:788-91.

4. Ruemmele FM, Targan SR, Levy G, et al. Diagnostic accuracy of serological assays in pediatric inflammatory bowel disease. Gastroenterology 1998;115:822-9.

5. Koutroubakis IE, Petinaki E, Mouzas IA, et al. Anti-Saccharomyces cerevisiae mann antibodies and antineutrophil cytoplasmic autoantibodies in Greek patients with inflammatory bowel disease. Am J Gastroenterol 2001;96:449-54.

6. Vasiliauskas EA, Plevy SE, Landers CJ, et al. Perinuclear antineutrophil cytoplasmic antibodies in patients with Crohn's disease define a clinical subgroup. Gastroenterology 1996;110:1810-9.

7. Duerr RH, Targan SR, Landers CJ, et al. Netrophil cytoplasmic autoantibodies: A link between PSC and UC. Gastroenterology 1991;100:1385-91.

8. Sandborn WJ, Landers CJ, Tremaine WJ, et al. Association of antineutrophil cytoplasmic antibodies with resistance to treatment of left-sided ulcerative colitis: Results of a pilot study. Mayo Clin Proc 1996;71:431-6.

9. Esteve M, Mallolas J, Klaassen J, et al. Antineutrophil cytoplasmic antibodies in sera from colectomised ulcerative colitis patients and its relation to the presence of pouchitis. Gut 1996;38:894-8. 


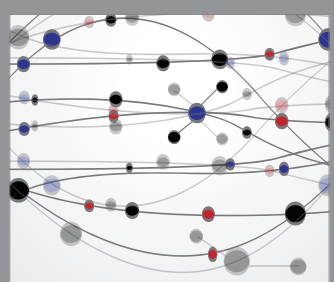

The Scientific World Journal
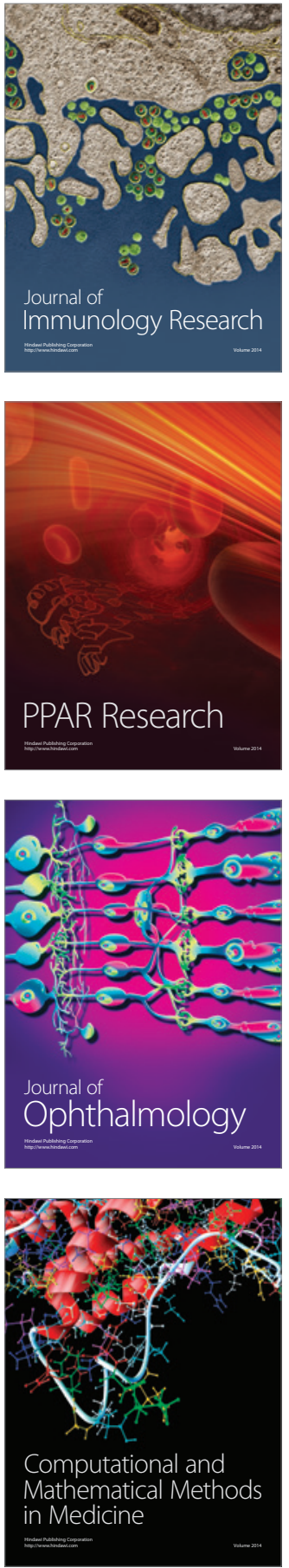

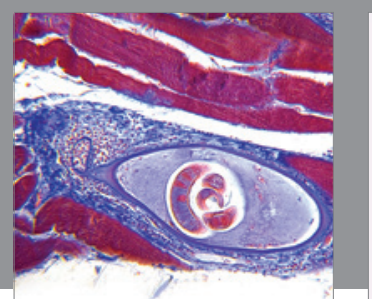

Gastroenterology Research and Practice

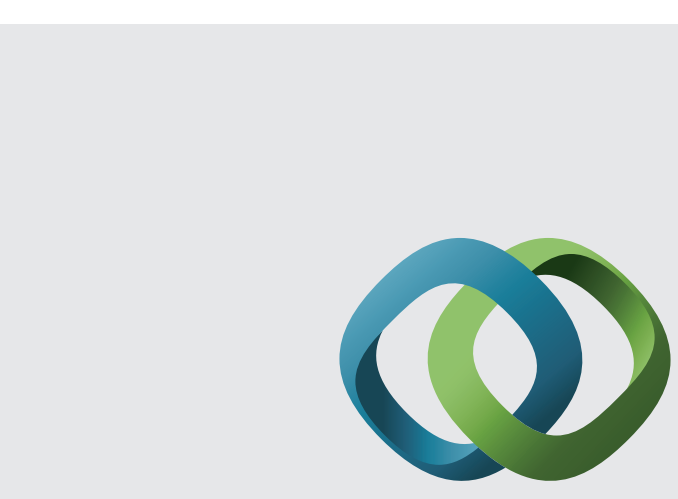

\section{Hindawi}

Submit your manuscripts at

http://www.hindawi.com
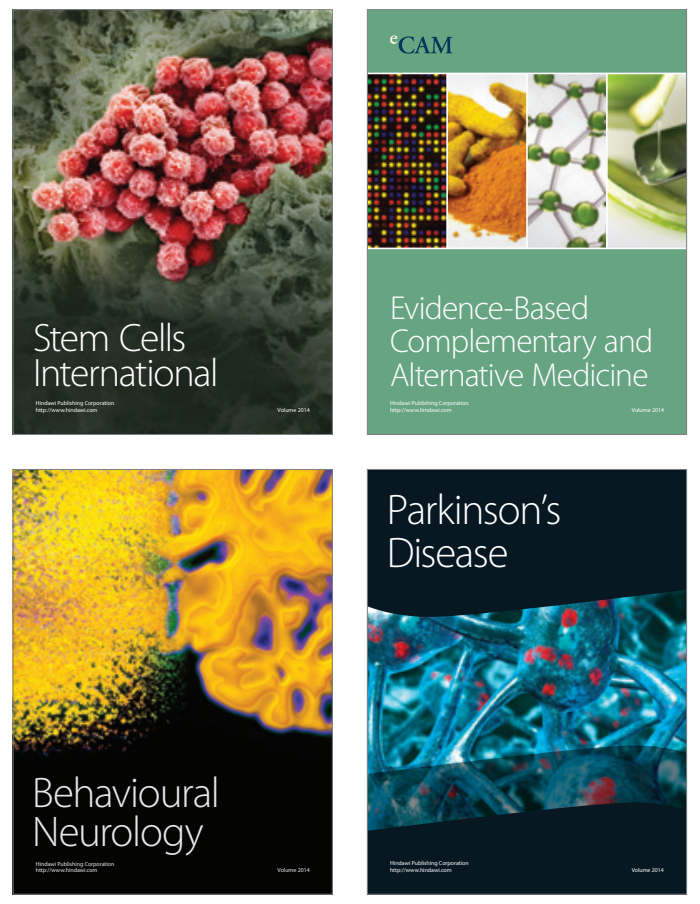
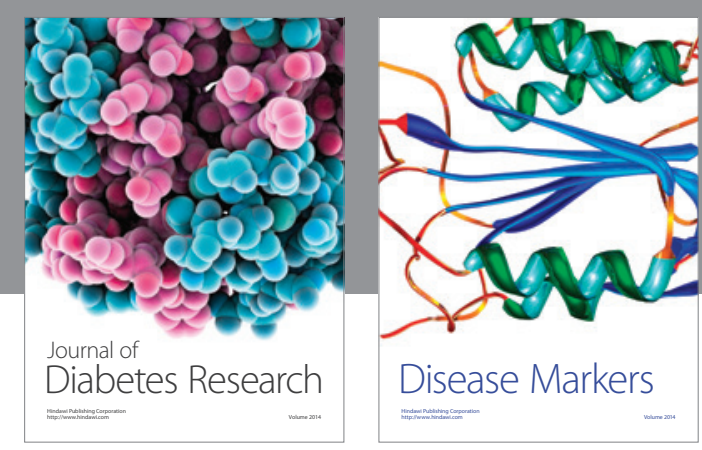

Disease Markers
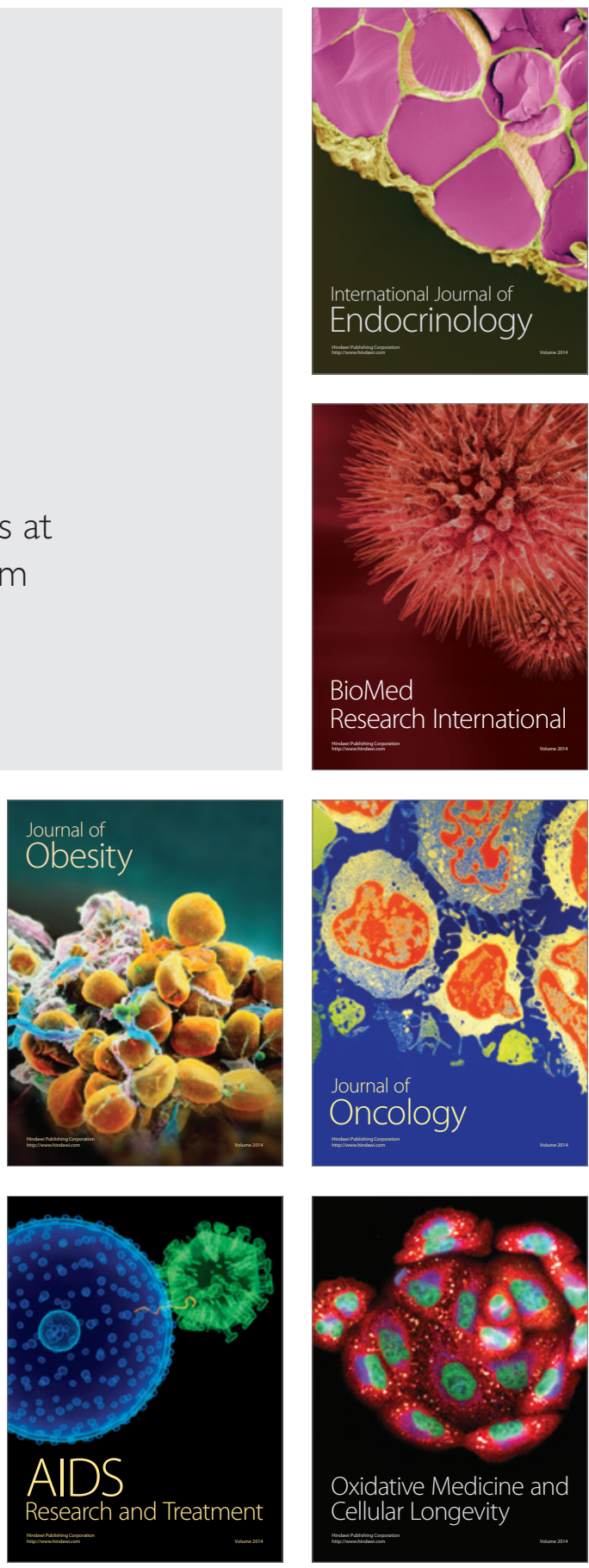